\title{
GENE SEGREGATION IN DIPLOIDISED AUTOTETRAPLOIDS
}

\author{
D. J. HARBERD \\ Department of Plant Sciences, University of Leeds
}

Received 11.iii.75

LADIZINSKI (1973) has recently published evidence that in the Avena strigosa complex the tetraploid $A$. barbata, which is bivalent forming at meiosis, is essentially the autotetraploid form of the diploid $A$. strigosa. The autotetraploid of $A$. strigosa obtained by colchicine treatment of the diploid is however quadrivalent forming, and this anomaly is accounted for by the finding that $A$. strigosa carries a dominant gene $A$ which permits full pairing between homologues, whereas $A$. barbata is aaa with pairing limited to bivalents. Under this system the triploid interspecific hybrid is trivalent forming; and duplex tetraploids derived from crosses between $A$. barbata and autotetraploid $A$. strigosa segregate on selfing to give 35 quadrivalent forming: 1 bivalent forming.

Several other workers have shown that natural tetraploids can be both bivalent forming and essentially autotetraploid, e.g. Lovis (1964), Mulligan (1967), Harberd and McArthur (1972), though there are many other equally or less convincing claims. There is, however, a natural reluctance to accept " bivalent forming autotetraploids" as a common phenomenon in plants, stemming from two main sources. Firstly, the almost universal observation that artificial autotetraploids (in which there can be no doubt that the material is autotetraploid) are quadrivalent forming. Secondly, the absence of any known mechanism for the restriction of pairing in autotetraploids to bivalents, apart from reduction of chiasma frequency (Dawson, 1941) which, other than in Lotus, is normally an insufficient explanation. Ladizinski's work does not solve this second problem but, taken to its logical conclusion, it does pose it in a slightly different way. Is it possible to visualise a mechanism whereby a material completely autotetraploid and with a high chiasma frequency is nevertheless bivalent forming at meiosis? Random pairing is presumably excluded since along with the high chiasma frequency this should lead to multivalents. Thought on specific pairing has generally concentrated on some form of chromosome differentiation, which is also excluded under the terms posed. One possible means of achieving bivalent formation in such a case would be for all pairing to be allosyndetic, that is for no two chromosomes which entered the zygote in the same gamete to be capable of pairing, even though homologous.

A restriction of chromosome pairing of this kind would have an effect on gene segregation in bivalent-forming autotetraploids. It would have no observable effect on segregations from triplex or simplex individuals, but duplex would differ according to whether they had originated from the fusion of (i) one gamete of $B B$ with one of $b b$, or (ii) two gametes of $B b$. In the former case all bivalents would be of $B$ with $b$, the gametes would be $1 B B: 2 B b: 1 b b$, and the resulting zygote segregation on selfing, $15: 1$. In the latter case gametes are expected in the proportions $1 B B: 6 B b: 1 b b$, giving a zygotic segregation $63: 1$ on selfing. Thus in neither case does 
the segregation resemble the $35: 1$ anticipated in the absence of the aaaa mechanism.

This note is prompted by the realisation that I have in fact published segregation ratios both of $15: 1$ and of $63: 1$ on selfing supposed autotetraploids with, at the time, no satisfactory explanation (Harberd and Watson, 1962). One plant of Agrostis canina tetraploid segregated on selfing 663 green : 10 albino, which is a close fit to $63: 1$ and significantly different from any other intelligible ratio. It was noted, furthermore, that this plant had an unusually high pollen fertility, which with hindsight might have been due to the fact that it was bivalent forming though this was never checked. More normal $A$. canina with the lower pollen fertility typical of this quadrivalent forming species segregated with ratios expected for duplex tetraploids. In the same paper we noted four plants of the tetraploid form of Festuca ovina which segregated in the $15: 1$ ratio. At the time we were concerned that this evidence, normally taken to indicate an allotetraploid segregation, was at variance with the bulk of evidence suggesting that tetraploid forms of the species are autotetraploid. It is now suggested that in the presence of a mechanism of the type envisaged, $15: 1$ ratios can be obtained from autotetraploids, and that therefore this evidence need not contradict the belief that $F$. ovina tetraploids are autotetraploid.

\section{REFERENCES}

DAwson, C. D. R. 1941. Tetrasomic inheritance in Lotus corniculatus L. F. Genet., 42, 49-72. HARBERD, D. J., AND MCARTHUR, E. D. 1972. Cyto-taxonomy of Rhynchosinapis and Hutera (Cruciferae-Brassicae). Heredity, 28, 254-257.

HARBERD, D. J., AND WATSON, P. J. 1962. A note on segregation ratios in tetraploid grasses. Scottish Plant Breeding Station Record for 1962, 58-60.

LADIZINSKI, G. 1973. Genetic control of bivalent pairing in the Avena strigosa polyploid complex. Chromosoma, 42, 105-110.

Lovis, J. D. 1964. Autopolyploidy in Asplenium. Nature, 203, 324-325.

Mulligan, G. A. 1967. Diploid and autotetraploid Physaria vitulifera (Cruciferae). Can. 7 . Bot., 45, 183-188. 